\title{
The use of confocal Raman spectroscopy to characterise the microstructure of complex biomaterials: foods
}

\author{
Paul D.A. Pudney ${ }^{\mathrm{a}, *}$, Thomas M. Hancewicz ${ }^{\mathrm{b}}$ and Dale G. Cunningham ${ }^{\mathrm{a}}$ \\ ${ }^{a}$ Unilever Bestfoods R\&D, Colworth Laboratory, Sharnbrook, Bedford MK44 1LQ, UK \\ ${ }^{\mathrm{b}}$ Unilever R\&D US Edgewater Laboratory, Edgewater, NJ, USA
}

\begin{abstract}
The properties and behaviour of many biomaterials often depends crucially on their microstructure. This is especially true of the largest class of biomaterials in use, foods. They include general properties, e.g., food texture, and others, such as spreadability of margarine/butter, pourablity of ketchup, scoopablity of ice cream, and also flavour release (a problem that has much in common with drug delivery), to name but a few. Thus, most food laboratories do a large amount of work in rheology and microscopy to relate structure and properties. However, a knowledge gap exists, i.e., what is the location and quantity of ingredients/molecules within a structure? Both of these questions need to be answered if a complete understanding is to be obtained. The "what is where" question can sometimes be answered by using various microscopic labelling techniques, although there can be many problems with these methods. Bulk separation is often attempted, followed by some kind of more classical analysis, but this is often either not possible or may cause some kind of uncontrolled perturbation. Thus, the ability to obtain quantitative information in situ within a microstructure has been an unobtained goal in much of food science research. These types of questions are, of course, also asked in many related fields of research.

This paper will illustrate how the advances in confocal Raman spectroscopy have allowed this problem to be tackled, in a noninvasive way. It will show how careful development of experimental procedures and advances in data analysis methods, allow even quantitative maps of microstructures to be obtained. The details of this approach will be described including a discussion of the limitations of current methods, especially depth resolution, and how these were overcome. The use of these methods will be illustrated with a gelled mixture of two carbohydrate polymers, $\kappa$-carageenan and gellan. Despite being similar polymers, and hence having highly overlapping spectra, the pure spectral components can be separated using a chemometrics based method, multivariate curve resolution (MCR). The principles of this method will be described. Under certain concentration regimes these two biopolymers phase separate. This property can be used to produce different microstructures. Two different microstructures were produced and mapped using Raman spectroscopy. This data was analysed using the MCR method to show the relative locations of the two polymers within the microstructure. Furthermore, by augmenting the data from these maps with calibration data for the two bioploymers, quantitative maps were produced. The resultant concentrations can then be used to produce tie lines for the $\kappa$-carageenan/gellan phase diagram, which is essential for understanding and manipulating the structure and properties in a systematic way. This methodology can be readily extended to much more complex multicomponent systems, such as complete food products.

This paper shows that the combination of the confocal Raman spectroscopy and MCR data analysis methodology is very powerful and is readily applicable in many areas of research, especially the biomaterial/biomedical fields.
\end{abstract}

\section{Introduction}

As has been recognised for some considerable time, Raman spectroscopy has a number of advantages over conventional techniques when looking at biological systems. Like its sister technique of infrared spectroscopy, it is highly sensitive to molecular species, but, unlike infrared spectroscopy, it is relatively insensitive to water, allowing it to be more easily applied in biology's predominant solvent. A Raman

\footnotetext{
${ }^{*}$ Corresponding auhor. Tel.: +44 1234 222486; Fax: +44 1234 222757; E-mail: Paul.Pudney@ unilever.com.
} 
spectrum is usually obtained through inelastic scattering of coherent monochromatic light from a laser, so that it can be focussed on a small spot. Thus it has long been recognised as having potential as a microscopic spectroscopic technique, which can be also made confocal (unlike infrared spectroscopy, which cannot). However, in the past, its application has been limited for a number of reasons. These included its relative lack of sensitivity, which often required prohibitively long data collection times, and the fluorescence background that is present in many biological/food systems. In recent years technological advances in Raman systems, such as new optical elements and improvements in detector technology that have increased sensitivity, have largely overcome these drawbacks. New, longer wavelength, stable lasers are now also available that allow the majority of the fluorescence signal to be avoided (this can also be achieved by using UV lasers).

These advances have lead to an increased use of Raman microspectroscopy in many areas. In biological systems sub-cellular structures in both animals [1] and plants [2] have been successfully Raman mapped. In material/polymer science Raman is widely used to map the relative spatial location and concentration of polymers [3]. So Raman appears to be closer to reaching its potential in this area. However, for it to become more generally applicable and more widely accepted, there are still problems to be overcome. The most common and easiest way to analyse a Raman map or image is to find a characteristic strong peak in each component's spectrum that is also free from interference from spectra of the other components present. This can be problematical because in a lot of systems, especially "real-life" multicomponent ones, locating single bands for components free from interference can be difficult, if not impossible. Also in a lot of systems, the identity of the components that are actually present may be unknown. How then are the relevant bands to be chosen? This problem is especially acute in biological systems where many components will come form the same molecular class. Thus their spectra, although different, are likely to be strongly overlapping. The approach we have taken to overcome this problem is to explore chemometic methods. This has lead to development and use of a number of data resolution/unmixing methods called self-modelling curve resolution. One of these methods in particular, multivariate curve resolution (MCR), has been effectively applied to the analysis of Raman image data [4]. This method allows even very similar spectra to be separated without prior knowledge of the components identities. Images or maps for all statistically significant chemicals/components present can thus be produced. Furthermore, this method has now been extended so that quantitative concentration maps can be produced by adding the appropriate calibration data. In this paper, to show how effective this method can be, we have examined a gelled microstructure of two carbohydrate biopolymers. These biopolymers, gellan and $\kappa$-carageenan, have highly overlapping spectra. A suitable part of the observed microstructure was Raman mapped and concentration calibration data collected for both polymers, this allowed concentration maps to be produced for both polymers. This data could then be compared with the optically observed microstructure and part of the phase diagram for the two polymers obtained.

Spatial resolution is another major issue in Raman imaging that is often overlooked, leading to erroneous interpretation of results. This is most commonly because, as has recently been pointed out by Everall [5,6], the standard confocal Raman set-up, i.e., a metallurgical objective, with the laser traversing an interface that has a refractive index change, gives rise to spherical aberration. This will distort the laser focus spot size in the $z$-plane; thus the spatial resolution will not then be at the specification of the instrument. This effect has been examined experimentally and showing that careful consideration of the experimental set-up can minimise this effect. 


\section{Experimental}

The studies were carried out using a Kaiser holoprobe 5000R confocal Raman spectrometer with $785 \mathrm{~nm}$ laser excitation. This is a Raman spectrometer optically coupled to an Olympus BX60 via optical fibres. The Raman spectrometer was chosen for its high sensitivity (most biopolymers have a low Raman scattering cross section) and stability (for quantitative work). It has a holographic notch filter, and grating, which give a high signal throughput to the detector. The detector is also liquid nitrogen cooled with a deep depleted back-thinned CCD array for high sensitivity. There is a pinhole placed between the spectrometer and the microscope so the Raman signal is collected confocally (N.B. the optical view using the microscope itself is not confocal). The spatial resolution using a 15 micron pinhole, on a planar interface in air, is $1.9 \mu \mathrm{m}$ in the $x-y$ plane and $2.9 \mu \mathrm{m}$ in $z$ plane. The depth resolution $(z)$ however can be affected by the sample/sampling conditions; this is discussed later in the paper. Appropriate spectral variables are given with the results.

\section{Data analysis methods}

Although Raman spectroscopy is a non-linear process, Raman spectral intensities are directly proportional to concentration within the relatively small deviation in Raman response in typical analytical experiments. This also assumes that no other changes in the system and samples are occurring. Thus the standard way to measure concentration with Raman spectroscopy is to choose a peak that is free from interference, and calibrate its intensity for absolute concentration against the corresponding Raman peak from the spectrum of a reference material of known concentration. As described in the introduction this method has a number of problems, particularly because of overlapping peaks. In the past this has meant that although information my have been inherent in the spectra, in practice it has been impossible to extract. This has lead to the exploration of chemometrics methods. The problem described here is lends itself most to the use of data resolution/unmixing methods called self-modelling curve resolution. One of these methods in particular, multivariate curve resolution (MCR) has been effectively applied to the analysis of Raman image data. The details of MCR have been given elsewhere [4] so only the pertinent details are given here.

The aim of the analysis of a large spectral data set from a multicomponent system (e.g., a Raman map of a microstructure) is to separate the total spectral response into two factor modes, one describing the pure spectra and one describing the pure intensities. In practice these correspond to the real spectra of the components present in the data and (at this stage) the uncalibrated concentration of each component for each spectrum. To do this, firstly a Principal Factor Analysis (PFA) is performed on the unscaled data. PFA gives a number of factors and scores. The PFA factors and scores are linear combinations of the pure spectral components and their intensities. The aim now is to "unmix" the factors and scores into pure "spectral" factors and "concentration" scores. To achieve this the PFA factors and scores are transformed by means of a constrained least-squares minimisation (LS) process. A number of constraints can be used, but in this problem the most appropriate are non-negativity and equality. The non-negativity constraint is necessary to push the LS result in a direction that is meaningful, given the nature of the original data. In this case having a negative Raman band in a spectrum or intensity (and hence concentration) makes no physical sense. So this constraint is applied alternatively to the factors and scores until a solution converges. The equality constraint is a normalisation of the factors after each iteration. This forces the factors (spectra) to be basis vectors, and thus forces all the variance into the intensity factor mode. 
This is required when quantitative information is desired because all variation in the data should be a function of concentration alone. The spectral profile therefore should contain no intensity information. The normalisation constraint on the factors helps to achieve this result. The MCR solution thus gives the pure factors (spectra) and scores (intensities), as long as you have chosen the correct number of factors. Choosing the correct number of factors is another complex issue and will not be discussed here; see work by Malinowski [7-9]. The resulting scores at this stage of the analysis give relative concentrations, i.e., a score value that goes from 1 to 2 shows a doubling of concentration, but not absolute values. To make the results fully quantitative calibration spectra have to be acquired for the components of interest (preferably separately and as miscible mixtures). These spectra are then added to the data set before performing MCR data analysis. The MCR analysis will then produce calibrated intensity scores (i.e., concentration values) which are then used to calibrate the response for the original Raman spectra. This method can thus be used to produce calibrated concentration maps from spectral maps of a microstructure.

This MCR method has a number of advantages over the more traditional single peak intensity calibration. The separation into pure spectral factors removes most of the problems associated with background changes causing errors, which often occur when measuring single peak intensities, as well as removing the problem of overlapping peaks. As the MCR method also uses the whole spectrum, it effectively increases the signal-to-noise ratio as compared with a single peak, thus reducing the error. The MCR method is also applicable to much more complex mixtures of many components. The method as developed and applied here to concentration measurements is described more fully elsewhere $[10,11]$.

\section{Spatial resolution}

Spatial resolution is another major issue. As stated in the experimental section, the spatial resolution of the instrumental set-up, as used in these experiments is $1.9 \mu \mathrm{m}$ in the $x-y$ plane and $2.9 \mu \mathrm{m}$ in the $z$-plane, on a planar interface in air. However the spatial resolution is defined as the distance it takes for a signal to decrease to half the maximum (assuming a point source or perfect plane interface). Thus if concentrations are required then it is necessary for the sample volume to be larger than this to get an accurate valve. The actual volume will depend on the accuracy required, but as previously estimated for infrared spectroscopy [12] it will be $2 / 3$ times the spatial resolution. Thus as the $z$-resolution is limiting in this case, a particle will need to be approximately $10 \mu \mathrm{m}$, given no other influences. However, it has recently been pointed out $[5,6]$ that, with the standard confocal Raman set-up, i.e., a metallurgical objective, and with the laser going through an interface that has a refractive index change, spherical aberration will occur, and this will distort the laser focus spot size in the $z$-plane. As the spatial resolution is a combination of the laser focus spot size and the confocal aperture, this will lead to degraded resolution. This has been calculated for a typical case with the laser going from air into a medium with refractive index of 1.5 [5]. The calculations show that the laser spot is greatly elongated inside the sample, and gets worse the deeper the laser is focussed. There is also the apparent depth effect to be considered, i.e., the laser appears to at a different depth inside the sample than it actually is, this can be a problem when trying to measure the size of structures in the $z$ dimension. However this result does not show the effect of the confocal pinhole. To perform a full analysis that includes the confocal pinhole is very complex, and depends on the exact instrumental set-up. Batchelder et al. [13] have performed a good approximate analysis for a similar set-up to the one used here. The results show that the confocal pinhole supplies a substantial improvement, but the depth resolution is still much lower and still gets worse deeper into the sample. This analysis shows that it is better to measure close to the interface (in this case the cover slip). The 


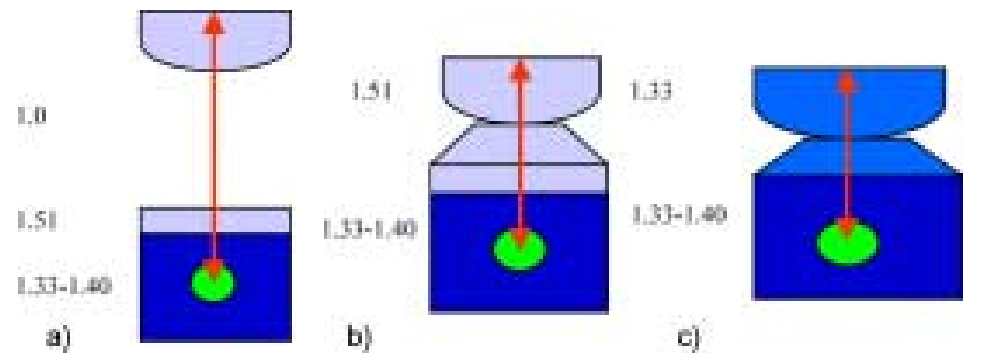

Fig. 1. Schematic diagram showing the various optical set-ups. (a) Normal Raman set-up with metallurgical objective, (b) oil immersion objective, (c) water immersion objective. The refractive index of each medium is labelled.

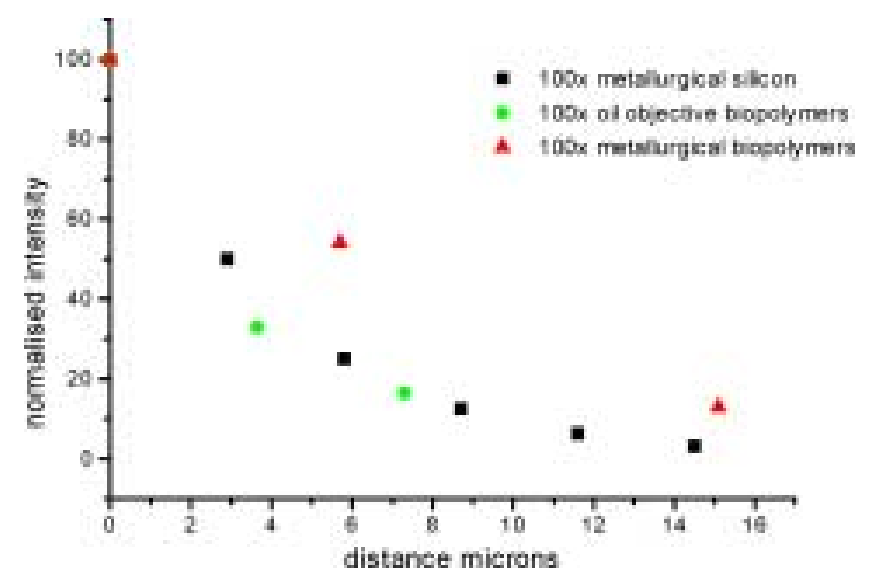

Fig. 2. Graph showing the effect on depth resolution of different optical set-ups.

calculations give the $z$-spatial resolution to be approx. $6.5 \mu \mathrm{m}$ at 20 microns depth through the interface. This would mean that one would need a particle of approximately 20 microns depth to get acceptable accuracy for concentration measurements.

However, one way to further improve resolution exists, which is the use of index matched objectives. The alternatives are either an oil immersion objective matched to the coverslip, or a water immersion objective directly placed onto the sample. These set-ups are shown in Fig. 1. These have been tested on a model system. The test system used was microgel particles of $10 \%$ agarose in $2 \%$ xanthan solution. The laser was then focussed into the centre of particles of different sizes, and the Raman spectrum collected. These spectra were then analysed to see how much signal (if any) was observable from the surrounding xanthan. A plot of the normalised intensities from the xanthan against distance to the xanthan, i.e., particle radius, is shown in Fig. 2. This shows that the $100 \times$ metallurgical objective (N.A. 0.8) used in this study has a depth resolution of $6.4 \mu \mathrm{m}$, the same, within error, as the calculated value by Batchelder [13]. The $100 \times$ oil objective (N.A. 1.25) has a resolution of $2.2 \mu \mathrm{m}$ (similar to that observed on a silicon wafer in air with the $100 \times$ metallurgical objective, also plotted in Fig. 2); this is significantly better resolution, and will allow particles that are less than $10 \mu \mathrm{m}$ to be examined with good accuracy in the concentration values. As expected, it was difficult in practise to use the water immersion objective, especially with small particles, because, as the objective has to touch the sample it can also move particles in the suspension. However, no xanthan could be detected when focusing in a $20 \mu \mathrm{m}$ agarose particle, showing it also has 
significantly better depth resolution than the metallurgical objective. Thus, when good spatial resolution is needed, experiments should be carried using an objective index matched to the sample.

\section{Results}

A phase separate gelled microstructure was investigated to show how the Raman technique could be applied to structures on the micron length scale. Additionally, this system would help in trying to understand the limitations of the methodology and how these limitations might be overcome. A carbohydrate/carbohydrate system was chosen, as these components are very similar biopolymers chemically and hence spectroscopically, thus showing the universality of the method to all biopolymer mixtures, no matter how similar. Raman spectra of the two, as pure gels, are shown in Fig. 3. The spectra are highly overlapping and it would not possible to use single peaks to measure the concentration of each biopolymer when mixed. Two concentration mixtures that phase separated were examined. They were $2 \% / 2 \%$ and $3 \% / 3 \%(\mathrm{w} / \mathrm{w})$ in $0.1 \mathrm{M} \mathrm{KCl}$. These were produced by mixing in solution at $100^{\circ} \mathrm{C}$ and then cooling quiescently. As it does so the mixture phase separates, giving the microstructure observed in Figs 5 and 6. Then, various $x-y$ Raman maps were collected through the centre of phase separated particles and the surrounding matrix, using a $100 \times$ oil objective. A range of calibration standards was acquired for both the pure gelled components and mixtures. The number of mixtures was limited by the fact that they must be in the miscible region of the phase diagram. The calibration gave an $R^{2}$ of 0.993 for carrageenan and 0.982 for gellan. The calibration data was then added to the mapping data and analysed using the MCR method. The factors for both gellan and $\kappa$-carrageenan separated well and are compared with the pure component spectra in Fig. 4. The typical calibrated score maps, i.e., concentration maps, are plotted in Figs 5 and 6, along with the optical pictures of the structure mapped.

From these quantitative maps, concentrations at the end of tie-lines were obtained. This was done by averaging the concentrations over a small area in the centre of a phase separated particle and an area of the matrix phase free from particles. For the $2 \% / 2 \%$ system the particle contains $4.2 \%( \pm 0.1)$ gellan and $0.7 \%( \pm 0.1) \kappa$-carrageenan and the matrix has $0.8 \%( \pm 0.1)$ gellan and $2.6 \%( \pm 0.15) \kappa$-carrageenan. The $3 \% / 3 \%$ system has $4.8 \%( \pm 0.1)$ gellan and $1.3 \%( \pm 0.1) \kappa$-carrageenan in the phase separated

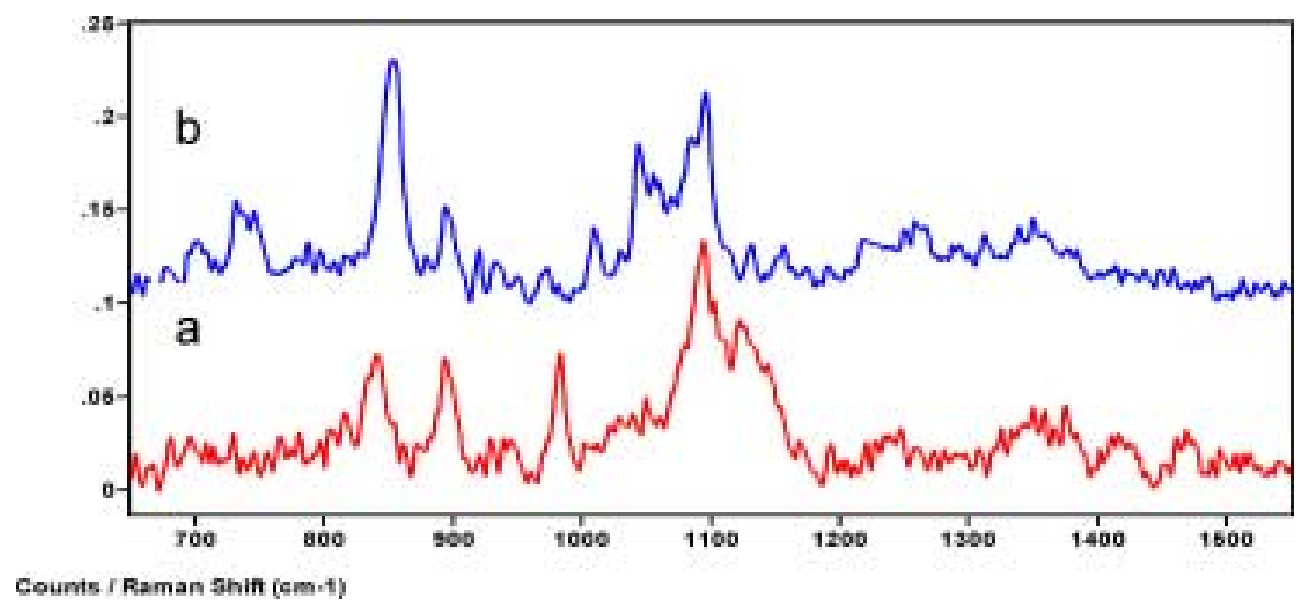

Fig. 3. Raman spectra of (a) pure gellan gel, (b) pure $\kappa$-carageenan gel. $100 \mathrm{~s}$ collection time. 


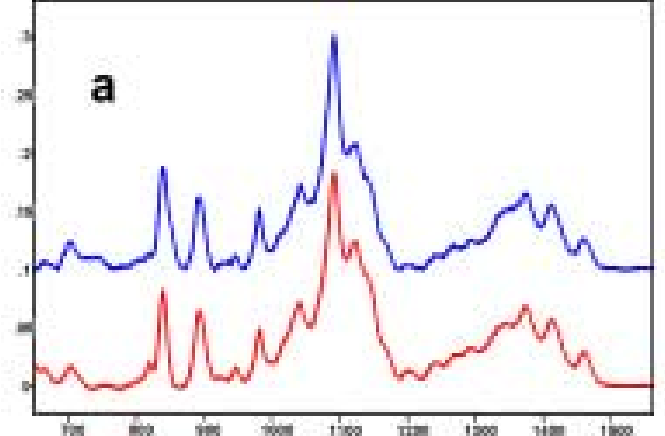

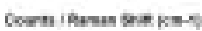

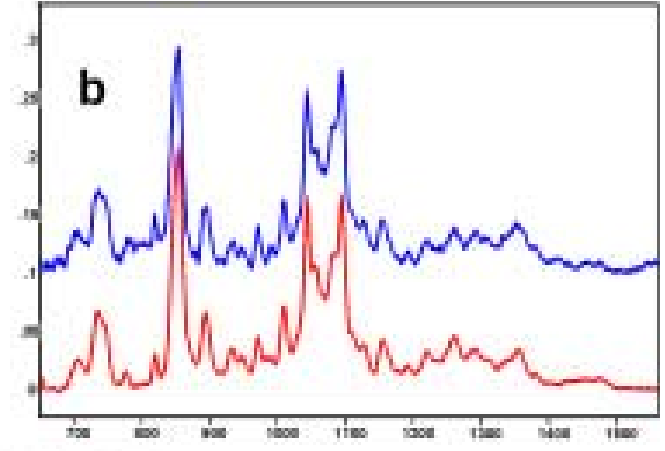

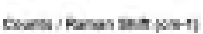

Fig. 4. MCR factors (red) and Raman spectra (blue) (a) gellan, (b) $\kappa$-carrageenan. Rescaled and offset for comparison.

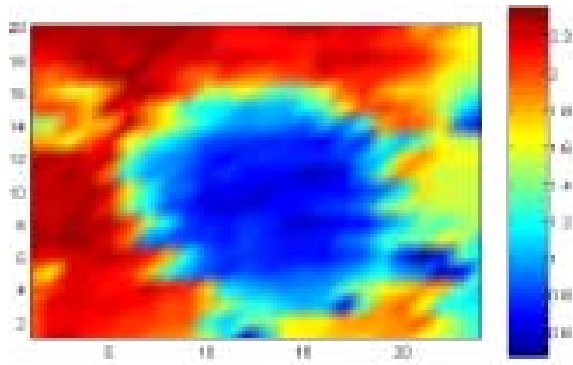

a

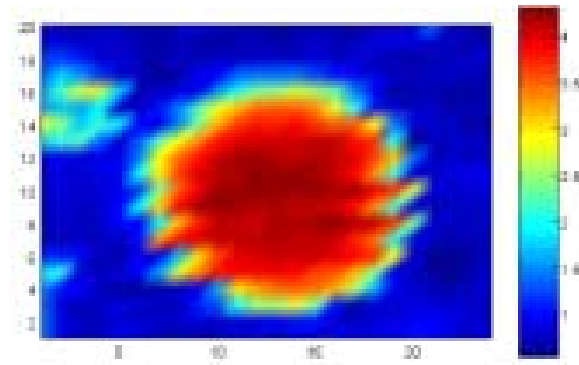

b

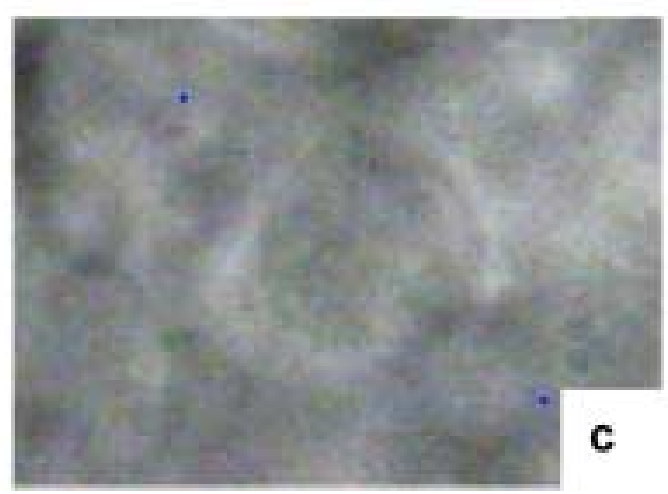

Fig. 5. Concentration maps of (a) $\kappa$-carrageenan, (b) gellan produced from Raman mapping the phase separated area shown in (c) of $2 \% \kappa$-carrageenan/2\% gellan. 480 spectra ( 24 by 20 ) were collected for $100 \mathrm{~s}$ each, at $1 \mu \mathrm{m}$ stepsize.

particle and $1.3 \%( \pm 0.1)$ gellan and $4.2 \%( \pm 0.25) \kappa$-carrageenan in the matrix, for more details see reference [10].

The phase diagram that is obtained from data like these is the starting point for the systematic manipulation of the material properties of a mixed biopolymer system. Also, concentration values from within a microstructure are needed when trying to understand various properties of materials. In the case of food bioploymer mixtures, understanding the mechanical properties and rheology is often very important, and an example where Raman obtained concentration values were essential has been shown previously [14]. 


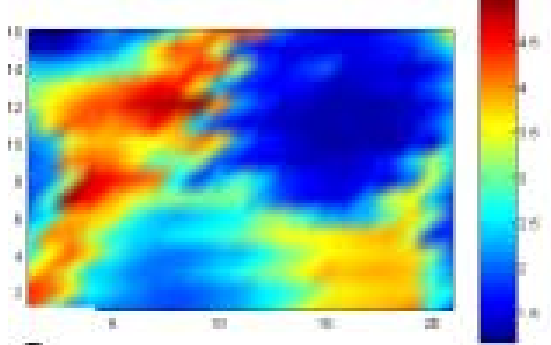

a

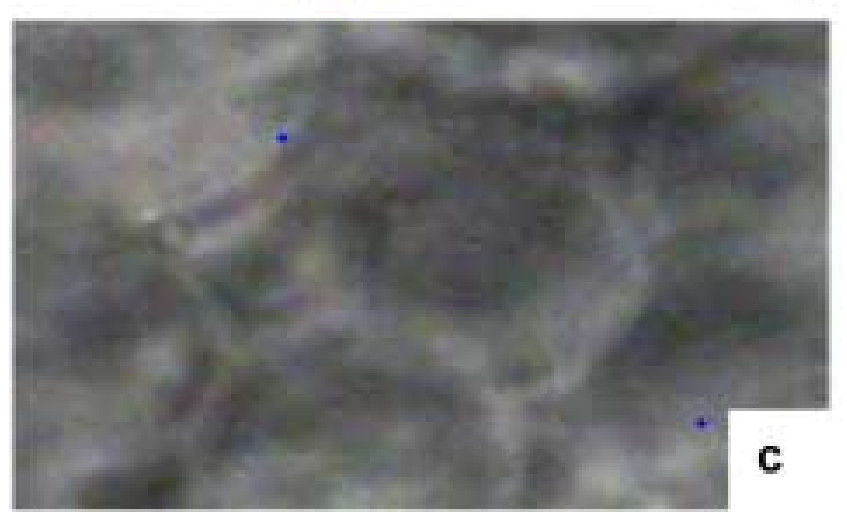

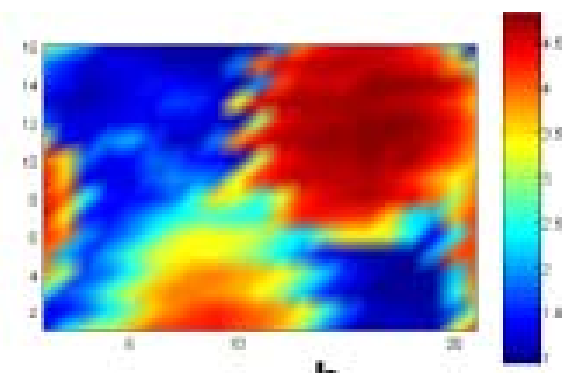

b

Fig. 6. Concentration maps of (a) $\kappa$-carrageenan, (b) gellan produced from Raman mapping the phase separated area shown in (c) of 3\% $\kappa$-carrageenan/3\% gellan. 336 spectra (21 by 16) were collected for $100 \mathrm{~s}$ each, at $1 \mu \mathrm{m}$ stepsize.

\section{Conclusion}

It has been shown that Confocal Raman microspectroscopy can be readily applied to characterise complex biomaterials. The developments that have been made in data analysis methods allow the untangling of spectra of even very similar biopolymers in complex mixtures, demonstrated in this case for a mixture of gellan and carrageenan. This allows images for all components to be produced and compared to the optically observed structure. Furthermore, it has been demonstrated that these component maps can be made quantitative. The spatial resolution of the method was examined experimentally and it was shown that great care is needed in choosing the experimental set-up to index match the spectrometer objective with the sample. If this is achieved a spatial resolution of 2 microns in the limiting $z$-axis is readily achievable. Thus accurate concentration valves are obtainable in structures of less than 10 microns.

\section{References}

[1] C. Otto, C.J. de Grauw, J.J. Duindam, N.M. Sijtsema and J. Greve, Applications of micro-Raman imaging in biomedical research, J. Raman Spectro. 28 (1997), 143.

[2] P.D.A. Pudney, L. Gambelli and M., Gidley, Raman mapping of plant cells:carotenoids in tomotoes, in: Proceedings 17th Conference on Raman Spectroscopy, 2000, 1076 ISBN 0471490075.

[3] K.P.J. Williams and N.J. Everall, Use of micro-Raman spectroscopy for the quantitative-determination of polyethylene density using partial least-squares calibration, J. Raman Spectro. 26 (1995), 427.

[4] J.J. Andrew and T.M. Hancewicz, Rapid analysis of Raman image data using two-way multivariate curve resolution, Appl. Spectro. 52 (1998), 797.

[5] N.J. Everall, Modelling and measuring the effect of refraction on the depth resolution of confocal Raman microscopy, Appl. Spectro. 54 (2000), 773. 
[6] N.J. Everall, Confocal Raman microscopy: Why the depth resolution and spatial accuracy can be much worse than you think, Appl. Spectro. 54 (2000), 1515.

[7] E.R. Malinowski, Chemometrics: Theory and applications, in: ACS Symp. Ser. 52, B.R. Kowalski, ed., American Chemical Society, Washington, DC, 1977, Chap. 3.

[8] E.R. Malinowski, Determination of number of factors and experimental error in a data matrix, Anal. Chem. 49 (1977), 612.

[9] E.R. Malinowski, Theory of the distribution of error eigenvalues resulting from principal component analysis with applications to spectroscopic data, J. Chemometrics 1 (1987), 33.

[10] P.D.A. Pudney, T.M. Hancewicz, D.G. Cunningham and C. Gray, A novel method for measuring concentrations of phase separated bioploymers: The use of confocal Raman spectroscopy with self-modelling curve resolution, Food Hydrocolliods, submitted.

[11] T.M. Hancewicz and P.D.A. Pudney, paper in prep.

[12] C.M. Durrini and A.M. Donald, Compositional mapping of mixed gels using FTIR microspectroscopy, Carbohydrate Polymers 289 (1995), 297.

[13] K.J. Baldwin and D.N. Batchelder, Confocal Raman microspectroscopy through a planar interface, Appl. Spectro. 55 (2001), 517

[14] V. Normand, P.D.A. Pudney, P. Aymard and I.T. Norton, Weighted-average isostrain and isostress model to describe the kinetic evolution of the mechanical properties of a composite gel: Application to the system gelatin : maltodextrin, J. Appl. Polymer Sci. 77 (2000), 1465. 


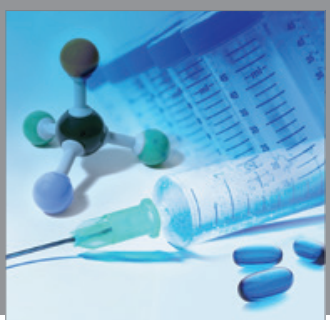

International Journal of

Medicinal Chemistry

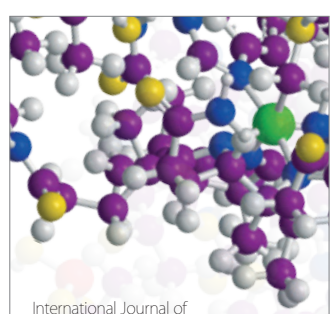

Carbohydrate Chemistry

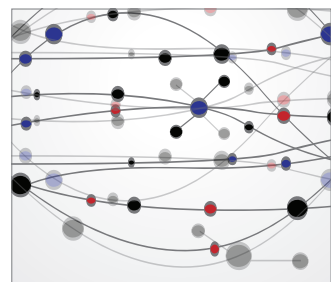

The Scientific World Journal
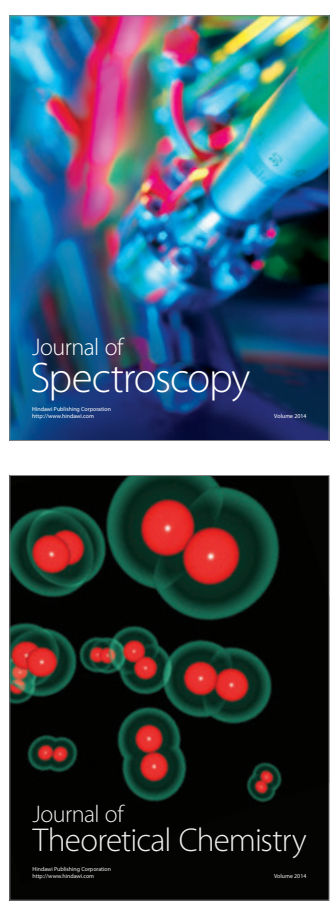
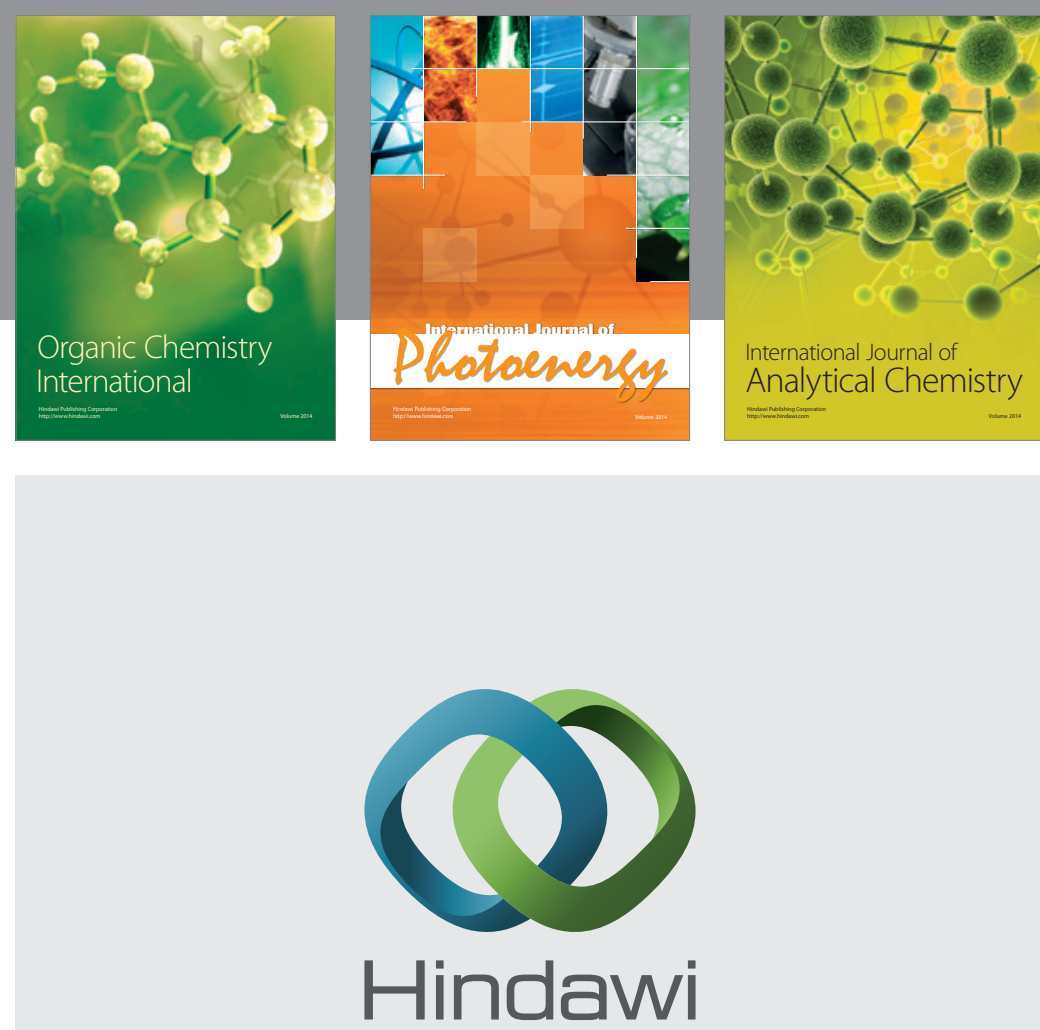

Submit your manuscripts at

http://www.hindawi.com
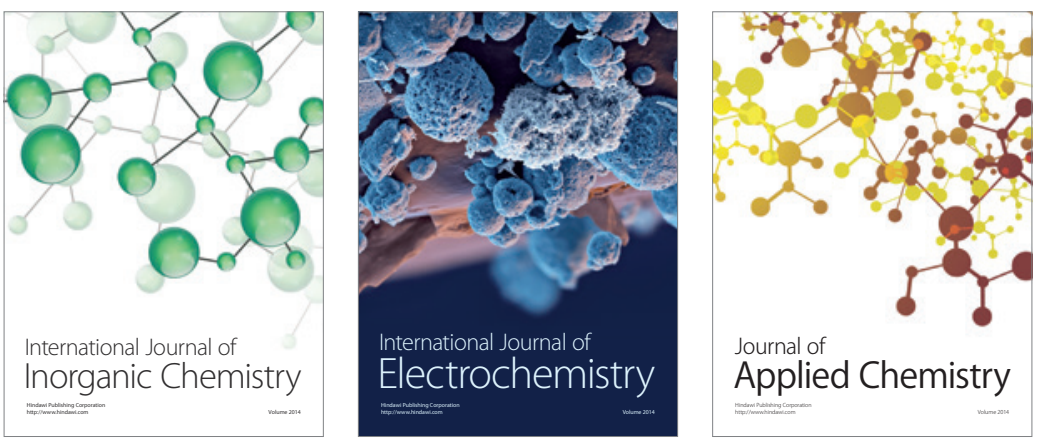

Journal of

Applied Chemistry
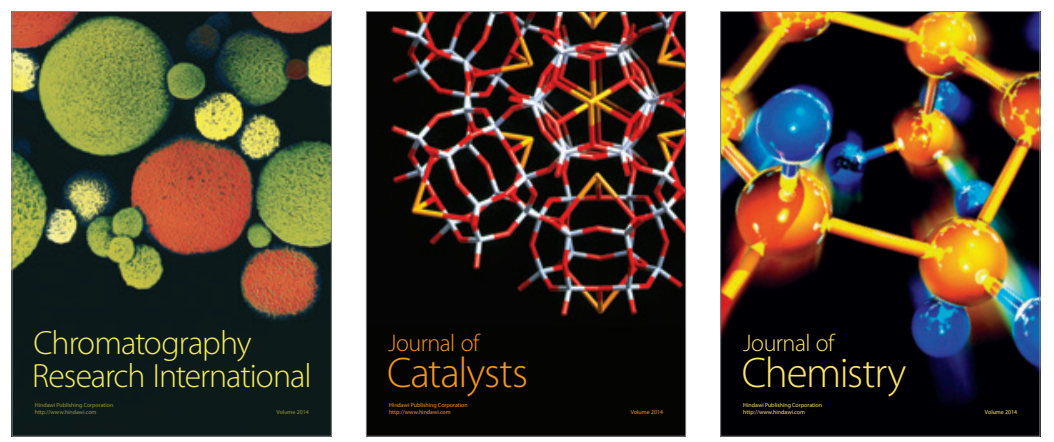
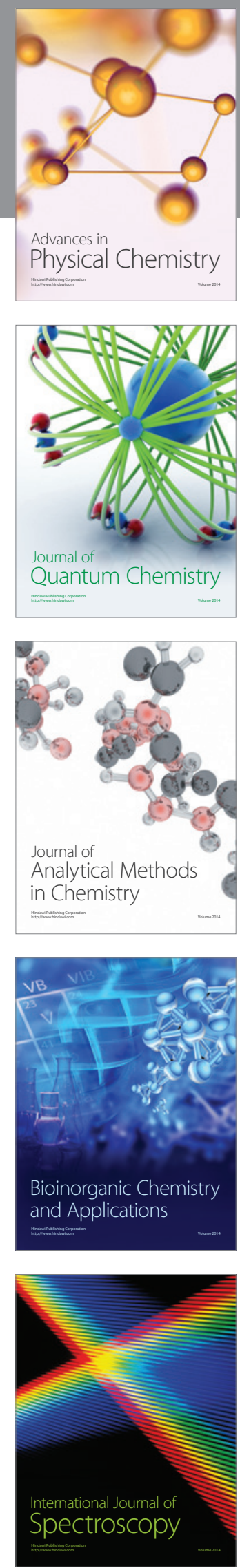